\title{
Invariant manifolds in dissipative dynamical systems*
}

\author{
Ferdinand Verhulst \\ Mathematisch Instituut \\ University of Utrecht \\ PO Box 80.010, 3508 TA Utrecht \\ The Netherlands
}

\begin{abstract}
Invariant manifolds like tori, spheres and cylinders play an important part in dynamical systems. In engineering, tori correspond with the important phenomenon of multi-frequency oscillations. Normal hyperbolicity guarantees the robustness of these manifolds but in many applications weaker forms of hyperbolicity present more realistic cases and interesting phenomena. We will review the theory and present a number of examples using normalization-averaging techniques.
\end{abstract}

\section{Introduction}

When studying dynamical systems, either generated by maps, ordinary differential equations, partial differential equations or other deterministic systems, a basic approach is to locate and to characterize the classical ingredients of such systems. These ingredients are critical points (equilibrium solutions), periodic solutions, invariant manifolds (in particular quasi-periodic tori), homoclinics, heteroclinics and in general stable and unstable manifolds of special solutions.

Here we will discuss invariant manifolds like slow manifolds, tori, cylinders with emphasis on the dissipative case. Consider a system like

$$
\dot{x}=f(x)+\varepsilon R(t, x, \varepsilon)
$$

${ }^{*}$ Dedicated to nonlinear scientist Aleš Tondl on his 80th birthday. To be publ. in Acta Applicandae Mathematicae, 2005. 
where $\varepsilon$ will indicate a small, positive parameter, $R$ represents a smooth perturbation. Suppose for instance that we have found an isolated torus $T_{a}$ by first order averaging or another normalizing technique. Does this manifold persist, slightly deformed as a torus $T$, when considering the original equation? Note that the original equation can be seen as a perturbation of an averaged or normalized equation and the question can then be rephrased as the question of persistence of the torus $T_{a}$ under perturbation.

If the invariant manifold in the averaged equation is normally hyperbolic the answer is affirmative (normally hyperbolic means loosely speaking that the strength of the flow along the manifold is weaker than the rate of attraction or repulsion to the manifold). We will discuss such cases. In many applications however the normal hyperbolicity is not easy to establish. In the Hamiltonian case the tori arise in families and they will not even be hyperbolic.

We will look at different scenarios for the emergence of tori in some examples. A torus is generated by various independent rotational motions - at least two - and we shall find different timescales characterizing these rotations.

Our emphasis on the analysis of invariant manifolds should be supplemented by appropriate numerical schemes. In Schilder, Osinga and Vogt (2004) continuation of quasi-periodic invariant tori is studied with a discussion of an algorithm, examples and extensive references. Another important aspect is the break-up, or more in general the bifurcations of tori. We will briefly discuss some of the results in this rich field. Bifurcations of invariant manifolds invoke much more complicated dynamics than bifurcations of equilibria or periodic solutions and there are still many problems to study.

\section{Deforming a normally hyperbolic manifold}

Consider the dynamical system in $\mathbb{R}^{n}$ described by the equation

$$
\dot{x}=f(x)
$$

and assume that the system contains a smooth $\left(C^{r}\right)$ invariant manifold $M$. The smoothness enables us to define a tangent bundle $T(M)$ and a normal bundle $N(M)$ of $M$. A typical situation in mechanics involves $N$ coupled two-dimensional oscillators containing a $m$-dimensional torus where $2 \leq m \leq N$. In this case $n=2 N$, the tangent bundle is $m$-dimensional, the normal bundle $(2 N-m)$-dimensional.

Hyperbolicity is introduced as follows. Assume that we can split the cor- 
responding normal bundle of $M$ with respect to the flow generated by the dynamical system in an exponentially stable one $N^{s}$ and an exponentially unstable one $N^{u}$ with no other components. In differential geometric terms the flow near the invariant manifold $M$ takes place on

$$
N^{s} \oplus T(M) \oplus N^{u} .
$$

In this case the manifold $M$ is called hyperbolic. If this hyperbolic splitting does not contain an unstable manifold $N^{u}, M$ is stable. For a more detailed discussion of these classical matters see for instance Hirsch, Pugh and Shub (1977).

Note that the smoothness of $M$ is needed in this description. In many cases the manifolds under consideration loose smoothness at certain bifurcation points when varying parameters. In such cases Lyapunov exponents can still be used to characterize the stability.

The manifold $M$ is moreover normally hyperbolic if, measured in the matrixand vector norms in $\mathbb{R}^{n}, N^{u}$ expands more sharply than the flow associated with $T(M)$ and $N^{s}$ contracts more sharply than $T(M)$ under the flow.

A number of details and refinements of the concept can be found in Hirsch, Pugh and Shub (1977), see also Shub (1987), Broer, Osinga and Vegter (1997).

Interestingly the concept of normal hyperbolicity is used often without explicit definition or even mentioning the term but is implicitly present in the conditions. Normal hyperbolicity in the case of a smooth manifold can be checked in a relatively simple way, in the case of nonsmoothness we have to adapt the definition.

In many applications the situation is simpler because a small parameter is present which induces slow and fast dynamics in the dynamical system. Consider the system

$$
\begin{aligned}
\dot{x} & =f(x, y), \quad x \in D \subset \mathbb{R}^{n}, t \geq 0 \\
\varepsilon \dot{y} & =g(x, y), \quad y \in G \subset \mathbb{R}^{m}
\end{aligned}
$$

with $f$ and $g$ sufficiently smooth vector functions in $x, y$. Putting $\varepsilon=$ 0 we have from the second equation $0=g(x, y)$ which we assume to be solvable by $\bar{y}=\phi(x)$ where $\phi(x)$ is a continuous function and an isolated root corresponding with a compact manifold. Fenichel (1971-79) has shown that if this root is hyperbolic, it corresponds with a nearby hyperbolic invariant manifold of the full system, a so-called slow manifold. In the analysis the fact that if this root is hyperbolic the corresponding manifold is also normally hyperbolic is inherent in the problem formulation. For the fibers of the slow 
manifold are ruled by the fast time-scale $1 / \varepsilon$ corresponding with a boundary layer in time while the dynamics of the drift along the manifold is ruled by the time-scale $O(1)$.

It is nearly trivial but useful to rewrite the system in different time-like variables. Putting $\tau=t / \varepsilon$ the system becomes

$$
\begin{aligned}
& \frac{d x}{d \tau}=\varepsilon f(x, y), \\
& \frac{d y}{d \tau}=g(x, y) .
\end{aligned}
$$

The slow variation of the variable $x$ which is $O(\varepsilon)$ is set off against the fast motion of $y$ which varies $O(1)$.

A simple example of a normally hyperbolic torus with small perturbations is the system

\section{Example 2.1}

$$
\begin{aligned}
\ddot{x}+x & =\mu\left(1-x^{2}\right) \dot{x}+\varepsilon f(x, y), \\
\ddot{y}+\omega^{2} y & =\mu\left(1-y^{2}\right) \dot{y}+\varepsilon g(x, y)
\end{aligned}
$$

with $\varepsilon$-independent positive constant $\omega$ and $\mu$ (fixed positive numbers, $O(1)$ with respect to $\varepsilon$ ) and smooth perturbations $f, g$. Omitting the perturbations $f, g$ we have two uncoupled normally hyperbolic oscillations. In general if $\omega$ is irrational the combined oscillations attract to a torus in 4-space, the product of the two periodic attractors, filled with quasi-periodic motion. Adding the perturbations $f, g$ can not destroy this torus but only deforms it. In this example the torus is two-dimensional but the timescales of rotation, if $\mu$ is large enough, are in both directions determined by the timescales of relaxation oscillation (see Grasman, 1987) and so are $O(1 / \mu)$.

There are natural extensions to non-autonomous systems by introducing the so-called stroboscopic map. We demonstrate this by an example derived from Broer, Osinga and Vegter (1997). See also the monograph by Broer, Huitema and Sevryuk (1996).

\section{Example 2.2}

Consider the forced van der Pol-oscillator

$$
\ddot{x}+x=\mu\left(1-x^{2}\right) \dot{x}+\varepsilon \cos \omega t
$$


which we write as the system

$$
\begin{aligned}
\dot{x} & =y, \\
\dot{y} & =-y+\mu\left(1-x^{2}\right) y+\varepsilon \cos \tau, \\
\dot{\tau} & =\omega .
\end{aligned}
$$

The $2 \pi$-periodic forcing term $\varepsilon \cos \tau$ produces a stroboscopic map of the $x, y$-plane into itself. For $\varepsilon=0$ this is just the map of the periodic solution of the Van der Pol-equation, an invariant circle, into itself and the closed orbit is normally hyperbolic. In the extended phase space $\mathbb{R}^{2} \times \mathbb{R} / 2 \pi \mathbb{Z}$ this invariant circle for $\varepsilon=0$ corresponds with a normally hyperbolic torus which is persistent for small, positive values of $\varepsilon$.

Actually, the authors, choosing $\mu=0.4, \omega=0.9$ consider what happens if $\varepsilon$ increases. At $\varepsilon=0.3634$ the normal hyperbolicity is destroyed by a saddle-node bifurcation.

\section{Slow manifolds}

Early approximation theory, in particular by Tikhonov is concerned with singular perturbation problems where the attraction, at least for some time, is directed towards the regular expansion of the solution with respect to the small parameter which corresponds with a stable critical point of the boundary layer equation. For a description and references see Verhulst (2005).

In the case of autonomous equations it is possible to associate with the regular asymptotic expansion a manifold in phase-space and to consider the attraction properties of the flow near this manifold. This raises the question whether these manifolds really exist or if they are just a phantom phenomenon.

Such questions were addressed and answered in a number of papers by Fenichel (1971, '74, '77, '79), Hirsch, Pugh and Shub (1977) and other authors; the reader is referred to the survey papers by Jones (1994), Kaper (1999), Kaper and Jones (2001).

Consider again the autonomous system

$$
\begin{aligned}
\dot{x} & =f(x, y)+\varepsilon \cdots, & x \in D \subset \mathbb{R}^{n}, \\
\varepsilon \dot{y} & =g(x, y)+\varepsilon \cdots, & y \in G \subset \mathbb{R}^{m} .
\end{aligned}
$$

As before $y$ is called the fast variable, $x$ the slow variable. The zero set of $g(x, y)$ is given by $\bar{y}=\phi(x)$ which in this autonomous case represents a first-order approximation $M_{0}$ of the $n$-dimensional (slow) manifold $M_{\varepsilon}$. The 
flow on $M_{\varepsilon}$ is to a first order approximation described by $\dot{x}=f(x, \phi(x))$. We assume hyperbolicity of the approximate slow manifold; in the constructions of a stable manifold we assume

$$
\text { Re Sp } g_{y}(x, \phi(x)) \leq-\mu<0, x \in D,
$$

i.e. the eigenvalues of the linearized flow near $M_{0}$, derived from the equation for $y$, have negative real parts only. In geometric singular perturbation theory, for which Fenichel's results are basic, we only assume that all real parts of the eigenvalues are non-zero. In this case the slow manifold $M_{\varepsilon}$ is normally hyperbolic.

The slow manifold being hyperbolic but unstable allows for interesting phenomena. One might approach $M_{\varepsilon}$ for instance by a stable branch, stay for some time near $M_{\varepsilon}$, and then leave again a neighbourhood of the slow manifold by an unstable branch. This produces solutions indicated as 'pulse-like', 'multi-bump solutions' etc. This type of exchanges of the flow near $M_{\varepsilon}$, is what one often looks for in geometric singular perturbation theory.

\section{Existence of the slow manifold}

The question whether the slow manifold $M_{\varepsilon}$ approximated by $\bar{y}=\phi(x)$ persists for $\varepsilon>0$ was answered by Fenichel. The main result is as follows:

If $M_{0}$ is a compact manifold which is normally hyperbolic, it persists for $\varepsilon>0$, i.e. there exists for sufficiently small, positive $\varepsilon$ a smooth manifold $M_{\varepsilon}$ close to $M_{0}$. Corresponding with the signs of the real parts of the eigenvalues there exist stable and unstable manifolds of $M_{\varepsilon}$, smooth continuations of the corresponding manifolds of $M_{0}$, on which the flow is fast. To a first order approximation the fast flow is described by the boundary layer equation

$$
\varepsilon \dot{y}=g(x(0), y)
$$

where the initial value is chosen outside the slow manifold, $y(0) \neq \phi(x(0))$. There are some differences between the cases where $M_{0}$ has a boundary or not. For details see Jones (1994), Kaper (1999) and the original papers.

\section{The compactness property}

Note that the assumption of compactness of $M_{0}$ is essential for the uniquess of the slow manifold. In many examples and applications $M_{0}$, the approximation of the slow manifold obtained from the fast equation, is not bounded. This can be remedied, admittedly in an artificial way, by applying a suitable cut-off of the vector field far away from the domain of interest. In this way compact domains arise which coincide locally with $D$ and $G$. However, this 
may cause some problems with the uniqueness of the slow manifold. We have for instance the following example:

\section{Example 3.1}

Consider the system

$$
\begin{aligned}
\dot{x} & =x^{2}, x(0)=x_{0}>0, \\
\varepsilon \dot{y} & =-y, y(0)=y_{0} .
\end{aligned}
$$

Putting $\varepsilon=0$ produces $y=0$ which corresponds with $M_{0}$. We can obtain a compact domain for $x$ by putting $l \leq x \leq L$ with $l$ and $L$ positive constants independent of $\varepsilon$. However, the limiting behaviour of the solutions depends on the initial condition. Integration of the phase-plane equation yields

$$
y(x)=y_{0} \exp \left(\frac{1}{\varepsilon x}-\frac{1}{\varepsilon x_{0}}\right) .
$$

As $x(t)$ increases (for $t=1 / x_{0}, x(t)$ becomes infinite) the solution for $y(t)$ tends to

$$
y_{0} \exp \left(-\frac{1}{\varepsilon x_{0}}\right)
$$

So the solutions are for $x(0)>0$ and after an initial fast transition all exponentially close to $y=0$. There are however an infinite number of slow manifolds dependent on $x_{0}$, all tunnelling into this exponentially small neighbourhood of $M_{0}$ given by $y=0$.

One might wonder about the practical use of exponential closeness as such solutions cannot be distinguished numerically. The phenomenon is important and of practical use when there is a change of stability or in general a bifurcation of the slow manifold. Also, exponentially close orbits may demonstrate very different sticking phenomena; see again Verhulst (2005).

\section{Example 3.2}

As a more complicated illustration we consider the van der Pol relaxation oscillator with parametric excitation

$$
\ddot{x}+x=\mu\left(1-x^{2}\right) \dot{x}+\mu c^{2} \dot{x} \cos ^{2} q t
$$

with $\mu \gg 1, c$ and $q$ positive constants. We write the equation as the autonomous system

$$
\begin{aligned}
\ddot{x}+x & =\mu\left(1-x^{2}\right) \dot{x}+\mu \dot{x} y^{2}, \\
\ddot{y}+q^{2} y & =0
\end{aligned}
$$


with initial values $y(0)=c, \dot{y}(0)=0$. Following Verhulst and Abadi (2005) we introduce the generalized Liénard transformation $(x, \dot{x}) \rightarrow(x, z)$ while putting $\varepsilon=1 / \mu$ to produce the equivalent system

$$
\begin{aligned}
\varepsilon \dot{x} & =z+x-\frac{1}{3} x^{3}+x y^{2}, \\
\dot{z} & =-x-2 x y \dot{y}, \\
\ddot{y}+q^{2} y & =0 .
\end{aligned}
$$

The approximate slow manifold $M_{0}$ is given by

$$
z=-x\left(1+y^{2}\right)+\frac{1}{3} x^{3}
$$

which corresponds with a 3 -dimensional cubic cylinder parallel to the $\dot{y}$-axis in 4-space. Note that $M_{0}$ is not compact which might imply non-uniqueness. The slow manifold is stable if $1-x^{2}+y^{2}<0$ on $M_{0}$, unstable if $1-x^{2}+y^{2}>0$. The possibility of stability changes invokes the presence of interesting limit sets. Numerical experiments show that these can be (multiple) relaxation oscillations with either a periodic or aperiodic character.

\section{Tori by Bogoliubov-Mitropolsky-Hale continua- tion}

The branching off of tori is more complicated than the emergence of periodic solutions in dynamical system theory. The emergence of tori was considered extensively by Bogoliubov and Mitropolsky (1961) using basically continuation of quasiperiodic motion under perturbations; for a summary and other references see also Bogoliubov and Mitropolsky (1963). Another survey together with new results can be found in Hale (1969); see the references there. A modern formulation in the more general context of bifurcation theory can be found in Chow and Hale (1982).

We present several theorems from Hale (1969) in an adapted form; see also Hale (1963).

Theorem 1

Consider the system $S$

$$
\begin{aligned}
\dot{\theta} & =\omega(t, \theta)+\varepsilon \omega_{1}(t, \theta, x, y)+\varepsilon^{2} \cdots, \\
\dot{x} & =A(\theta) x+\varepsilon A_{1}(t, \theta, x, y)+\varepsilon^{2} \cdots, \\
\dot{y} & =B(\theta) y+\varepsilon B_{1}(t, \theta, x, y)+\varepsilon^{2} \cdots
\end{aligned}
$$


with $\theta \in \mathbb{R}^{k}, x \in \mathbb{R}^{n}, y \in \mathbb{R}^{m}$; all vector functions on the righthand side are periodic in $\theta$ and $t$.

Such a system arises naturally from local perturbations of differential equations in a neighbourhood of an invariant manifold where the 'unperturbed' system

$$
\dot{\theta}=\omega(t, \theta), \dot{x}=A(\theta) x, \dot{y}=B(\theta) y
$$

is assumed to have an invariant manifold $M_{0}$ given by

$$
M_{0}=\{(t, \theta, x, y): x=y=0\} .
$$

We also assume for system $S$ that

1. All vector functions on the righthand side are continuous and bounded; the $\varepsilon^{2} \ldots$ terms represent vector functions which are smooth on the domain and which can be estimated $O\left(\varepsilon^{2}\right)$.

2. The functions on the righthand side are Lipschitz-continuous with respect to $\theta$, the function $\omega(t, \theta)$ with Lipschitz constant $L$.

3. The functions $A_{1}, B_{1}, \omega_{1}$ are Lipschitz-continuous with respect to $x, y$.

4. There exist positive constants $K$ and $\alpha$ such that for any continuous $\theta(t)$ the fundamental matrices of $\dot{x}=A(\theta) x, \dot{y}=B(\theta) y$ can be estimated by $K e^{-\alpha t}, K e^{\alpha t}$ respectively.

5. $\alpha>L$ (normal hyperbolicity)

then there exists an invariant manifold $M$ of system $S$ near $M_{0}$ with Lipschitzcontinuous parametrization which is periodic in $\theta$.

Note that although $\alpha$ and $L$ are independent of $\varepsilon$ the difference may be small. In the applications one should take care that $\varepsilon=o(\alpha-L)$.

Another remark is that Hale's results are much more general. For instance the vector functions need not be periodic in $\theta$ but only bounded. If the vector functions are almost periodic the parametrization of $M$ inherits almost periodicity.

Even more importantly the perturbations $\varepsilon A_{1}, \varepsilon B_{1}$ in the equations for $x$ and $y$ can be replaced by $O(1)$ vector functions. However, this complicates the conditions of the corresponding theorem. Also, to check the conditions in these more general cases is not so easy.

We turn now to a case arising often in applications. 


\section{The case of parallel flow}

In a number of important applications the frequency vector $\omega(t, \theta)$ of system $S$ is constant; this will cause the flow on $M_{0}$ to be parallel. In this case $L=0$ and the fifth condition of theorem 1 is automatically satisfied. In addition the case of parallel flow makes it easier to consider cases where the attraction or expansion is weak:

\section{Theorem 2}

Consider the system $S_{w}$

$$
\begin{aligned}
\dot{\theta} & =\omega+\varepsilon \omega_{1}(t, \theta, x, y)+\varepsilon^{2} \cdots, \\
\dot{x} & =\varepsilon A(\theta) x+\varepsilon A_{1}(t, \theta, x, y)+\varepsilon^{2} \cdots, \\
\dot{y} & =\varepsilon B(\theta) y+\varepsilon B_{1}(t, \theta, x, y)+\varepsilon^{2} \cdots
\end{aligned}
$$

with constant frequency vector $\omega$. As before this $t$ - and $\theta$-periodic system is obtained by local perturbation of an invariant manifold $M_{0}$ in the system

$$
\dot{\theta}=\omega, \dot{x}=\varepsilon A(\theta) x, \dot{y}=\varepsilon B(\theta) y
$$

for $x=y=0$. In the equations for $x$ and $y, A(\theta) x$ and $B(\theta) y$ represent the linearizations near $(x, y)=(0,0)$ so $A_{1}, B_{1}$ are $o(\|x\|,\|y\|)$. Assume that

1. All vector functions on the righthand side are continuous and bounded; the $\varepsilon^{2} \cdots$ terms represent vector functions which are smooth on the domain and which can be estimated $O\left(\varepsilon^{2}\right)$.

2. The functions on the righthand side are Lipschitz-continuous with respect to $\theta$, the function $\omega_{1}$ with Lipschitz constant $\eta$.

3. The functions $\omega_{1}, A_{1}, B_{1}$ are Lipschitz-continuous with respect to $x, y$.

4. There exist positive constants $K$ and $\alpha$ such that for any continuous $\theta(t)$ the fundamental matrices of $\dot{x}=\varepsilon A(\theta) x, \dot{y}=\varepsilon B(\theta) y$ can be estimated by $K e^{-\varepsilon \alpha t}, K e^{\varepsilon \alpha t}$ respectively.

5. $\alpha>\eta$ (normal hyperbolicity at higher order)

then there exists an invariant manifold $M$ of system $S_{w}$ near $M_{0}$ with Lipschitz-continuous parametrization which is periodic in $\theta$.

To facilitate the analysis we introduce a normal form technique based on averaging; for an introduction see Verhulst (2000). 
The frequency vector being constant in system $S_{w}$ enables us to introduce slowly varying phases by putting

$$
\theta(t)=\omega t+\psi(t)
$$

The resulting system $S_{w}$ is of the form

$$
\dot{X}=\varepsilon F(t, x)+\varepsilon^{2} \cdots,
$$

where we have replaced $(\psi, x, y)$ by $X$. The system is quasi-periodic in $t$. The near-identity transformation

$$
X(t)=z(t)+\varepsilon u(t, z(t)), u(t, z(t))=\int_{0}^{t}\left(F(t, z(t))-F_{0}(z(t))\right) d t
$$

with $F_{0}(z(t))$ the average over the periods of $F$ in $t$ leads to the equation

$$
\dot{z}=\varepsilon F_{0}(z)+\varepsilon^{2} \cdots .
$$

Note that as yet we have not introduced any approximation. Usually we can relate theorem 2 to the equation for $z$ which will in general - at least to $O(\varepsilon)$ - be much simpler than the system $S_{w}$.

We will present a few illustrative examples.

\section{Example 5.1}

Consider the system

$$
\begin{aligned}
\ddot{x}+x & =\varepsilon\left(2 x+2 \dot{x}-\frac{8}{3} \dot{x}^{3}+y^{2} x^{2}+\dot{y}^{2} x^{2}\right)+\varepsilon^{2} R_{1}(x, y), \\
\ddot{y}+\omega^{2} y & =\varepsilon\left(\dot{y}-\dot{y}^{3}+x^{2} y^{2}+\dot{x}^{2} y^{2}\right)+\varepsilon^{2} R_{2}(x, y) .
\end{aligned}
$$

$R_{1}$ and $R_{2}$ are smooth, bounded functions. This looks like a bad case: if $\varepsilon=0$ we have a family of (nonhyperbolic) 2-tori in 4-space. We introduce amplitude-angle coordinates by $x=r_{1} \cos \theta_{1}, \dot{x}=-r_{1} \sin \theta_{1}, y=$ $r_{2} \cos \omega \theta_{2}, \dot{y}=-\omega r_{2} \sin \omega \theta_{2}$. The system transforms to

$$
\begin{aligned}
\dot{\theta}_{1}= & 1-\varepsilon\left(2 \cos ^{2} \theta_{1}-\sin 2 \theta_{1}+\frac{8}{3} r_{1}^{2} \sin ^{3} \theta_{1} \cos \theta_{1}+\right. \\
& \left.r_{1} r_{2}^{2} \cos ^{3} \theta_{1}\left(\cos ^{2} \omega \theta_{2}+\omega^{2} \sin ^{2} \omega \theta_{2}\right)\right)+\varepsilon^{2} \cdots \\
\dot{\theta}_{2}= & 1+\varepsilon\left(\frac{1}{2 \omega} \sin \left(2 \omega \theta_{2}\right)+\omega r_{2}^{2} \sin ^{3} \omega \theta_{2} \cos \omega \theta_{2}-\frac{r_{1}^{2} r_{2}}{\omega^{2}} \cos ^{3} \omega \theta_{2}\right)+\varepsilon^{2} \cdots \\
\dot{r}_{1}= & \varepsilon\left(-r_{1} \sin 2 \theta_{1}+2 r_{1} \sin ^{2} \theta_{1}-\frac{8}{3} r_{1}^{3} \sin ^{4} \theta_{1}-\right. \\
& \left.r_{1}^{2} r_{2}^{2} \sin \theta_{1} \cos ^{2} \theta_{1}\left(\cos ^{2} \omega \theta_{2}+\omega^{2} \sin ^{2} \omega \theta_{2}\right)\right)+\varepsilon^{2} \cdots \\
\dot{r}_{2}= & \varepsilon\left(r_{2} \sin ^{2} \omega \theta_{2}+\omega^{2} r_{2}^{3} \sin ^{4} \omega \theta_{2}-\frac{r_{1}^{2} r_{2}^{2}}{\omega} \sin \omega \theta_{2} \cos ^{2} \omega \theta_{2}\right)+\varepsilon^{2} \cdots
\end{aligned}
$$


Putting $\theta_{1}=t+\psi_{1}, \theta_{2}=t+\psi_{2}$ and using the near-identity transformation introduced above but keeping - with some abuse of notation - the same symbols we find the much simpler system

$$
\begin{aligned}
& \dot{r}_{1}=\varepsilon r_{1}\left(1-r_{1}^{2}\right)+\varepsilon^{2} \cdots, \dot{\psi}_{1}=-\varepsilon+\varepsilon^{2} \cdots, \\
& \dot{r}_{2}=\varepsilon \frac{r_{2}}{2}\left(1-\frac{3}{4} r_{2}^{2}\right)+\varepsilon^{2} \cdots, \dot{\psi}_{2}=\varepsilon^{2} \cdots .
\end{aligned}
$$

The part of $(x, y)=(0,0)$ is played by $\left(r_{1}, r_{2}\right)=\left(1, \frac{2}{\sqrt{ } 3}\right)$. The averaged (normalized) equations contain a torus in phase-space approximated by the parametrization

$$
\begin{aligned}
& x_{a}(t)=\cos \left(t-\varepsilon t+\psi_{1}(0)\right), \dot{x}_{a}(t)=-\sin \left(t-\varepsilon t+\psi_{1}(0)\right), \\
& y_{a}(t)=\frac{2}{3} \sqrt{3} \cos \left(\omega t+\psi_{2}(0)\right), \dot{y}_{a}(t)=-\frac{2 \omega}{3} \sqrt{3} \sin \left(\omega t+\psi_{2}(0)\right) .
\end{aligned}
$$

From linearization of the averaged equations it is clear that the torus is attracting: it is normally hyperbolic with attraction rate $O(\varepsilon)$. If the ratio of $1-\varepsilon$ and $\omega$ is rational, the torus is filled up with periodic solutions. If the ratio is irrational we have a quasi-periodic (two-frequency) flow over the torus. Theorem 2 tells us that in the original equations a torus exists in an $O(\varepsilon)$ neighbourhood of the torus found by normalization. It has the same stability properties. The torus is two-dimensional and the timescales of rotation are in both directions $O(1)$.

In the next example we return to the forced van der Pol-equation from example 2.2 .

\section{Example 5.2}

Consider the equation

$$
\ddot{x}+x=\varepsilon\left(1-x^{2}\right) \dot{x}+a \cos \omega t
$$

with $a$ and $\omega$ constants. The difference with example 2.2 is that the nonlinearity is small and the forcing can be $O(1)$ as $\varepsilon \rightarrow 0$.

1. Case $a=O(\varepsilon)$.

If $\omega$ is $\varepsilon$-close to 1 , standard averaging leads to the existence of periodic solutions only. If $\omega$ takes different values first order averagingnormalization is not conclusive but see the remark below.

2. Case $a=O(1), \omega$ is not $\varepsilon$-close to 1 (if $\omega$ is near to 1 the solutions move away from an $O(1)$ neighbourhood of the origin because of linear 
resonance). We introduce the transformation $x, \dot{x} \rightarrow r, \psi$

$$
x=r \cos (t+\psi)+\frac{a}{1-\omega^{2}} \cos \omega t, \dot{x}=-r \sin (t+\psi)-\frac{a \omega}{1-\omega^{2}} \sin \omega t .
$$

The resulting slowly varying system can be averaged, producing periodic solutions in which various values of $\omega$ play a part. Returning to the corresponding expressions for $x$ and $\dot{x}$ we conclude to the presence of tori in the extended phase space.

Remark:

In some of the cases near-identity transformation leads to a slowly varying system of the form

$$
\begin{aligned}
\dot{r} & =\varepsilon \frac{1}{2} r\left(1-\frac{1}{4} r^{2}\right)+\varepsilon^{2} \cdots, \\
\dot{\psi} & =\varepsilon^{2} \cdots .
\end{aligned}
$$

Instead of computing higher order normal forms to establish the behaviour of $\psi$ we can apply slow manifold theory to conclude the existence of a slow manifold $\varepsilon$-close to $r=2$. In the case of $a=O(1)$ the corresponding solutions will be $\varepsilon$-close to the torus described by

$$
x=2 \cos \left(t+\psi_{0}\right)+\frac{a}{1-\omega^{2}} \cos \omega t, \dot{x}=-2 \sin \left(t+\psi_{0}\right)-\frac{a \omega}{1-\omega^{2}} \sin \omega t .
$$

\section{Tori created by Neimark-Sacker bifurcation}

Another important scenario to create a torus arises from the Neimark-Sacker bifurcation. For an instructive and detailed introduction see Kuznetsov (2004). Suppose that we have obtained an averaged equation $\dot{x}=\varepsilon f(x, a)$ with dimension 3 or higher by variation of constants and subsequent averaging; $a$ is a parameter or a set of parameters. It is well-known that if this equation contains a hyperbolic critical point, the original equation contains a periodic solution. The first order approximation of this periodic solution is characterized by the timescales $t$ and $\varepsilon t$.

Suppose now that by varying the parameter $a$ a pair of eigenvalues of the critical point becomes purely imaginary. For this value of $a$ the averaged equation undergoes a Hopf bifurcation producing a periodic solution of the averaged equation; the typical timescale of this periodic solution is $\varepsilon t$ and so the period will be $O(1 / \varepsilon)$. As it branches off an existing periodic solution in the original equation it will produce a torus; it is associated with a Hopf bifurcation of the corresponding Poincaré map and the bifurcation 
has a different name: Neimark-Sacker bifurcation. The result will be a two-dimensional torus which contains two-frequency oscillations, one on a timescale of order 1 and the other with timescale $O(1 / \varepsilon)$.

A typical example runs as follows.

\section{Example 6.1}

A special case of a system studied by Bakri et al. (2004) is

$$
\begin{aligned}
\ddot{x}+\varepsilon \kappa \dot{x}+(1+\varepsilon \cos 2 t) x+\varepsilon x y & =0, \\
\ddot{y}+\varepsilon \dot{y}+4(1+\varepsilon) y-\varepsilon x^{2} & =0 .
\end{aligned}
$$

This is a system with parametric excitation and nonlinear coupling; $\kappa$ is a positive damping coefficient which is independent of $\varepsilon$. Away from the coordinate planes we may use amplitude-phase variables by $x=r_{1} \cos (t+$ $\left.\psi_{1}\right), \dot{x}=-r_{1} \sin \left(t+\psi_{1}\right), y=r_{2} \cos \left(2 t+\psi_{2}\right), \dot{y}=-2 r_{2} \sin \left(2 t+\psi_{1}\right)$; after first order averaging we find, omitting the subscripts $a$, the system

$$
\begin{aligned}
\dot{r}_{1} & =\varepsilon r_{1}\left(\frac{r_{2}}{4} \sin \left(2 \psi_{1}-\psi_{2}\right)+\frac{1}{4} \sin 2 \psi_{1}-\frac{1}{2} \kappa\right), \\
\dot{\psi}_{1} & =\varepsilon\left(\frac{r_{2}}{4} \cos \left(2 \psi_{1}-\psi_{2}\right)+\frac{1}{4} \cos 2 \psi_{1}\right), \\
\dot{r}_{2} & =\varepsilon \frac{r_{2}}{2}\left(\frac{r_{1}^{2}}{4 r_{2}} \sin \left(2 \psi_{1}-\psi_{2}\right)-1\right), \\
\dot{\psi}_{2} & =\frac{\varepsilon}{2}\left(-\frac{r_{1}^{2}}{4 r_{2}} \cos \left(2 \psi_{1}-\psi_{2}\right)+2\right) .
\end{aligned}
$$

Putting the righthand sides equal to zero produces a nontrivial critical point corresponding with a periodic solution of the system for the amplitudes and phases and so a quasi-periodic solution of the original coupled system in $x$ and $y$.We find for this critical point the relations

$r_{1}^{2}=4 \sqrt{5} r_{2}, \cos \left(2 \psi_{1}-\psi_{2}\right)=\frac{2}{\sqrt{5}}, \sin \left(2 \psi_{1}-\psi_{2}\right)=\frac{1}{\sqrt{5}}, r_{1}=2 \sqrt{2 \kappa+\sqrt{5-16 \kappa^{2}}}$.

This periodic solution exists if the damping coefficient is not too large: $0 \leq$ $\kappa<\frac{\sqrt{5}}{4}$. Linearization of the averaged equations at the critical point while using these relations produces the matrix

$$
A=\left(\begin{array}{cccc}
0 & 0 & \frac{r_{1}}{4 \sqrt{5}} & -\frac{r_{1}^{3}}{40} \\
0 & -\kappa & \frac{1}{2 \sqrt{5}} & \frac{r_{1}^{2}}{80} \\
\frac{r_{1}}{4 \sqrt{5}} & \frac{r_{1}^{2}}{2 \sqrt{5}} & -\frac{1}{2} & -\frac{r_{1}^{2}}{4 \sqrt{5}} \\
-\frac{2}{r_{1}} & 1 & \frac{4 \sqrt{5}}{r_{1}^{2}} & -\frac{1}{2}
\end{array}\right) .
$$


Another condition for the existence of the periodic solution is that the critical point is hyperbolic, i.e. the eigenvalues of the matrix $A$ have no real part zero. It is possible to express the eigenvalues explicitly in terms of $\kappa$ by using a software package like MATHEMATICA. However, the expressions are cumbersome. Hyperbolicity is the case if we start with values of $\kappa$ just below $\frac{\sqrt{5}}{4}=0.559$. Diminishing $\kappa$ we find when $\kappa=0.546$ that the real part of two eigenvalues vanishes. This value corresponds with a Hopf bifurcation producing a nonconstant periodic solution of the averaged equations. This in its turn corresponds with a torus in the orginal equations (in $x$ and $y$ ) by a Neimark-Sacker bifurcation. As stated before, the result will be a two-dimensional torus which contains two-frequency oscillations, one on a timescale of order 1 and the other with timescale $O(1 / \varepsilon)$.

\section{$7 \quad$ Breakdown and bifurcations of tori}

Complementary to the emergence of tori, their breakdown is of great theoretical and practical interest. In particular we would like to have a general idea of how two-dimensional invariant tori break down and how nontrivial limit sets are created when certain parameters are varied. To obtain insight the analysis of maps can be very helpful as the phenomena governed by differential equations are much more implicit. We shall briefly discuss a few seminal papers playing a part in studies of this topic. More references can be found in these papers.

A common feature is the presence of stable and unstable periodic solutions in $p / q$-resonance on a torus. Breakup can be triggered by heteroclinic tangencies, arising when a parameter is varied. This leads rather quickly to strange, chaotic behaviour. There are other scenarios producing strange behaviour where the normal hyperbolicity of the torus decreases more gradually.

\subsection{Aronson et al. (1982)}

This tutorial paper is a very instructive introduction to the subject. The analysis centres around the two-dimensional map

$$
\begin{aligned}
x_{n+1} & =y_{n}, \\
y_{n+1} & =a y_{n}\left(1-x_{n}\right)
\end{aligned}
$$

and its two parameter unfolding

$$
(x, y) \longmapsto(y+b x, a y(1-x)) .
$$


However, apart from the technical details of this example, the general theory is explained throughout the paper. For certain parameter values the map with $b=0$ contains a smooth invariant circle. Changing the parameter the invariant circles grows and becomes eventually a strange attractor. A basic question is: what happens at this parameter value and what is the mechanism in the map dynamics to produce this transformation to chaotic behaviour.

A key idea is that resonance occurs on the invariant circle if there is a pair of periodic orbits, one stable and one unstable (sink and saddle). A $p / q$ resonance occurs if the rotation number of these periodic orbits is $p / q$ with $p$ and $q$ relative prime. The imbedding in the map with $b \neq 0$ helps to understand how these resonances are generated as resonance horns arise in parameter space; for details of this process see Kuznetsov (2004). When the invariant manifolds of periodic orbits start crossing in homoclinic or heteroclinic tangencies the invariant circle looses differentiability and chaotic dynamics takes over.

This is illustrated in great detail by computer assisted proofs for this map and its imbedding.

\subsection{Afraimovich and Shil'nikov (1991)}

This is a paper with stimulating ideas but with quite a few things to be filled in by the reader. Consider a smooth dynamical system governed by the equation $\dot{x}=X(x, \mu)$ where $\mu$ is a vector parameter. Assume that the system contains a smooth attracting torus for $\mu=\mu_{0}$ and no torus for $\mu=\mu_{1}\left(>\mu_{0}\right)$. Assume furthermore that for $\mu=\mu_{0}$ the torus contains two periodic orbits, one stable and one unstable (of saddle-type with a twodimensional unstable manifold), reflecting the $p / q$-resonance. Adding some minor assumptions and realising that breakdown of the torus starts with nonsmoothness of the torus, there are three possibilities:

1. The stable and unstable periodic orbits vanish through a bifurcation.

2. Stable and unstable manifolds of the unstable periodic orbit intersect tangentially to form a homoclinic orbit.

3. The stable periodic orbit looses stability.

The authors illustrate all three possibilities by the two-dimensional map

$$
\begin{aligned}
x_{n+1} & =\left(x_{n}+b \mu\left(1+a \sin \theta_{n}\right)\right)^{\nu}, \\
\theta_{n+1} & =\theta_{n}-\ln \left(x_{n}+b \mu\left(1+a \sin \theta_{n}\right)\right)
\end{aligned}
$$


with $0<x_{n} \leq x_{0}, \theta_{n} \in \mathbb{R}, \nu>1,0<\mu \leq \mu_{0}, 0<b<1,0 \leq a<1$.

\subsection{Broer, Simó and Tatjer (1998)}

This paper studies a rich dissipative family of maps of an annulus into itself, the so-called fattened Arnold map:

$$
\begin{aligned}
& x_{n+1}=x_{n}+\omega+\alpha\left(y_{n}+\sin x_{n}\right)(\bmod 2 \pi), \\
& y_{n+1}=\beta\left(y_{n}+\sin x_{n}\right)
\end{aligned}
$$

with real parameters $\omega, \alpha, \beta,\left(x_{n}, y_{n}\right) \in \mathbb{S}^{1} \times \mathbb{R}$.

If $\beta=0$ the circle $y=0$ is invariant producing the classical Arnold family of circle maps which models a basic scenario in mechanics. Using $\beta$ as a perturbation parameter the 'fattened' Arnold map emerges. The Jacobian of the map is $\beta$ and the paper is mainly concerned with the dissipative case $|\beta|<1$.

The detailed and informative analysis is based on analytic perturbation (normal form) techniques and on numerical bifurcation methods. As the parameters move one obtains a number of possible transitions from classical attractors to complex and chaotic dynamics. As expected one phenomenon here is the loss of smoothness of the invariant circle followed by its destruction. In addition there are homoclinic bifurcations, cascades of bifurcations, homoclinic and heteroclinic tangencies. Apart from 'small' strange attractors there exist 'large' strange attractors which are connected with homoclinic tangency.

\section{Acknowledgement}

References and comments on the manuscript by Taoufik Bakri, Henk Broer, Odo Diekmann and Hil Meijer are gratefully acknowledged.

\section{Literature}

1. Afraimovich, V.S. And Shil'Nikov, L.P. (1991), Invariant two-dimensional tori, their breakdown and stochasticity, Amer. Math. Soc, Transl. 149, pp. 201-212.

2. Aronson, D.G., Chory, M.A., Hall, G.R., McGehee, R.P. (1982), Bifurcations from an invariant circle for two-parameter families of maps of the plane: a computer-assisted study, Commun. Math. Phys. 83, pp. 303354 . 
3. Bakri, T,, Nabergoj, R., Tondl, A., Verhulst, F. (2004), Parametric Excitation in Nonlinear Dynamics, Int. J. Nonlinear Mech. 39, pp. 311-329.

4. Bogoliubov, N.N. And Mitropolsky, Yu.A. (1961), Asymptotic Methods in the Theory of Nonlinear Oscillations, Gordon and Breach, New York.

5. Bogoliubov, N.N. And Mitropolsky, Yu.A. (1963), The Method of Integral Manifolds in Nonlinear Mechanics, in Contributions to Differential Equations, vol.2, pp. 123-196, Interscience-John Wiley.

6. Broer, H.W., Huitema, G.B., Sevryuk, M.B. (1996), Quasi-periodic motions in families of dynamical systems: order amidst chaos, Lecture Notes Math. 1645, Springer-Verlag.

7. Broer, H.W., Osinga, H.M., Vegter, G. (1997), Algorithms for computing normally hyperbolic invariant manifolds, Z. angew. Math. Phys. 48, pp. $480-524$.

8. Broer, H.W., Simó, C., Tatjer, J.C. (1998), Towards global models near homoclinic tangencies of dissipative diffeomorphisms, Nonlinearity 11, pp. 667-770.

9. Chow, S.-N. And Hale, J.K. (1982), Methods of bifurcation theory, SpringerVerlag.

10. Fenichel, N. (1971), Persistence and smoothness of invariant manifolds for flows, Ind. Univ. Math. J. 21, pp. 193-225.

11. Fenichel, N. (1974), Asymptotic stability with rate conditions, Ind. Univ. Math. J. 23, pp. 1109-1137.

12. Fenichel, N. (1977), Asymptotic stability with rate conditions, II, Ind. Univ. Math. J. 26, pp. 81-93.

13. Fenichel, N. (1979), Geometric singular perturbations theory for ordinary differential equations, J. Diff. Eq. 31, pp. 53-98.

14. Grasman, J. (1987), Asymptotic methods for relaxation oscillations and applications, Appl.Math.Sciences 63, Springer-Verlag.

15. Hale, J.K. (1963), Oscillations in nonlinear systems, MacGraw-Hill, New York, repr. Dover Publ., New York (1992).

16. Hale, J.K. (1969), Ordinary Differential Equations, Wiley-Interscience.

17. Hirsch, M., Pugh, C., Shub, M. (1977), Invariant Manifolds, Lecture Notes in Mathematics 583, Springer Verlag, Heidelberg etc.

18. Jones, C.K.R.T. (1994), Geometric Singular Perturbation Theory, in Dynamical Systems, Montecatini Terme 1994 (R. Johnson, ed.), Lecture Notes Math. 1609, pp. 44-118, Springer-Verlag. 
19. KAPER, T.J. (1999), An introduction to geometric methods and dynamical systems theory for singular perturbation problems, Proc. Symposia Appl. Math, AMS 56: Analyzing multiscale phenomena using singular perturbation methods, (Jane Cronin and Robert E. O'Malley, Jr., eds.). pp. 85-131.

20. Kaper, T.J. And Jones, C.K.R.T. (2001), A primer on the exchange lemma for fast-slow systems, IMA volumes in mathematics and its applications 122: Multiple-Time-Scale Dynamical Systems, (C.K.R.T. Jones and A.I. Khibnik, eds.). Springer Verlag, New York.

21. Kuznetsov, Yu. A. (2004), Elements of applied bifurcation theory, $3^{d}$ ed., Springer-Verlag.

22. Schilder, F., Osinga, H.M., Vogt, W. (2004), Continuation of quasiperiodic invariant tori, preprint Bristol (see also the site www.enm.bris.ac.uk/anm/preprints).

23. Shub, M.. (1987), Global Stability of Dynamical Systems, Springer-Verlag.

24. Verhulst, F. (2000), Nonlinear Differential Equations and Dynamical Systems, Universitext, Springer-Verlag.

25. Verhulst, F. (2005), Methods and Applications of Singular Perturbations, boundary layers and multiple timescale dynamics, Springer-Verlag.

26. Verhulst, F. AND ABADI (2005), Autoparametric resonance of relaxation oscillations, to be publ. in ZAMM. 